\title{
High Serum Advanced Glycation End-Products Predict Coronary Artery Disease Irrespective of Arterial Stiffness in Diabetic Patients
}

\author{
Ki-Bum Won, MD ${ }^{1,2}$, Hyuk-Jae Chang, MD', Sung-Ha Park, MD1', \\ Sung-Yu Hong, PhD'1, Yangsoo Jang, MD', and Namsik Chung, MD' \\ 'Department of Cardiology, Yonsei Cardiovascular Center, Yonsei University College of Medicine, Seoul, \\ ${ }^{2}$ Department of Cardiology, Myongji Hospital Cardiovascular Center, Kwandong University College of Medicine, Goyang, Korea
}

Background and Objectives: Advanced glycation end-products (AGEs) contribute to the development of atherosclerosis. We investigated whether serum AGEs are related to the presence or severity of coronary artery disease (CAD), and explored the association between serum AGEs and arterial stiffness according to diabetes status in patients suspected of having CAD.

Subjects and Methods: The measurement of serum AGEs and brachial-ankle pulse wave velocity (baPWV) were performed in 145 consecutive patients ( $63 \pm 9$ years, $58 \%$ men) who received a coronary angiogram for evaluation of CAD.

Results: Forty-four diabetics and 101 non-diabetics were classified into three subgroups based on the number of diseased vessels with obstructive CAD: 0, 1, and 2 or more vessel diseases (VDs). Serum AGEs were significantly higher in diabetics with obstructive CAD than in those without obstructive CAD ( $2.16 \pm 0.29$ vs. $1.85 \pm 0.29 \mathrm{mU} / \mathrm{mL}, \mathrm{p}=0.010)$ and were significantly correlated with the number of VDs only in diabetics $(r=0.504, p<0.001)$. Serum AGEs were not significantly correlated with baPWV in diabetics or non-diabetics. In receiver operating characteristics analysis, the cut-off value of serum AGEs as a predictor of obstructive CAD was $1.98 \mathrm{mU} / \mathrm{mL}$, with $64 \%$ sensitivity and 63\% specificity in diabetics. In multiple regression analysis, serum AGEs independently predicted obstructive CAD and were associated with the number of VDs in diabetics.

Conclusion: Serum AGEs independently predict obstructive CAD and the severity of coronary atherosclerosis irrespective of arterial stiffness only in diabetics. Evaluation of PWV and serum AGEs together may be more effective to identify the risk of CAD in diabetic individuals.

(Korean Circ J 2012;42:335-340)

KEY WORDS: Advanced glycation end-products; Coronary artery disease; Pulse wave velocity; Diabetes mellitus.

\section{Introduction}

Cardiovascular diseases are a major cause of morbidity and mortality in patients with diabetes. ${ }^{1)}$ Conventional risk factors such as

Received: August 5, 2011

Revision Received: September 26, 2011

Accepted: October 26, 2011

Correspondence: Hyuk-Jae Chang, MD, Department of Cardiology, Yonsei Cardiovascular Center, Yonsei University College of Medicine, 50 Yonsei-ro, Seodaemun-gu, Seoul 120-752, Korea

Tel: 82-2-2228-8460, Fax: 82-2-393-2041

E-mail: hjchang@yuhs.ac

- The authors have no financial conflicts of interest.

This is an Open Access article distributed under the terms of the Creative Commons Attribution Non-Commercial License (http://creativecommons. org/licenses/by-nc/3.0) which permits unrestricted non-commercial use, distribution, and reproduction in any medium, provided the original work is properly cited. hypertension, dyslipidemia, and smoking are important in the development of diabetic cardiovascular complications, but they can explain only part of excess risk. Recent studies have suggested that hyperglycemia is also related to increased cardiovascular risk. ${ }^{2-4)}$ One of the potential mechanisms through which hyperglycemia may contribute to cardiovascular disease is through the formation of advanced glycation end-products (AGEs).

Advanced glycation end-products are bioactive molecules formed by non-enzymatic glycation of proteins, lipids and nucleic acid. AGEs increase in concentration with age and the generation of AGEs is accelerated by sustained hyperglycemia. ${ }^{5)}$ AGEs are related to increased arterial stiffness, which is an independent contributing factor for coronary artery disease (CAD), by inducing collagen cross-linking in the vessel wall.6) Increased arterial stiffness and decreased vascular distensibility lead to increased systolic pressure and cardiac afterload, and decreased diastolic blood pressure, which potentially 
compromises coronary blood flow. ${ }^{7 / 8)}$ AGEs also contribute to the development of atherosclerosis by binding to specific receptors, such as receptor for AGEs (RAGE), on endothelial cells.

Serum levels of AGEs are increased in both diabetic ${ }^{9) 10)}$ and nondiabetic ${ }^{11)}$ patients with CAD. Recently, elevated serum levels of AGEs have been reported to be associated with increased aortic stiffness in adults. ${ }^{12)}$ We postulated that serum levels of AGEs are useful for predicting the presence or severity of $C A D$, and elevated serum levels of AGEs are associated with increased aortic stiffness in patients suspected of having CAD. To address this hypothesis, we investigated whether serum levels of AGEs were different with and without $C A D$, and correlated serum levels of AGEs with brachial-ankle pulse wave velocity (baPWV), as a marker of arterial stiffness, according to the status of diabetes in patients with suspected of having CAD.

\section{Subjects and Methods}

\section{Study population}

We studied 145 consecutive patients, who had been referred to a tertiary referral hospital to be evaluated for suspected CAD. All subjects underwent an invasive coronary angiogram to verify the presence of obstructive CAD, and serum levels of AGEs and baPWV were also measured.

\section{Advanced glycation end-products}

Serum levels of AGEs were measured with a non-competitive enzyme-linked immunosorbent assay. A 96-well microplate was coated at $4^{\circ} \mathrm{C}$ overnight with serially diluted AGE-bovine serum albumin (BSA) as the standard, or diluted serum samples. Unbound sites were blocked with 2\% skim milk, 0.5\% BSA, and 0.5\% gelatin in $0.05 \mathrm{M}$ carbonate buffer for 1 hour and washed with phosphate buffered saline (0.05\% Tween-20). Each well was incubated for 2 hours with anti-AGE monoclonal antibody (dilution 1 : 1000; Transgenic, Kumamoto, Japan). The plate was washed again and incubated with horseradish peroxidase-labeled goat anti-mouse lgG antibody (dilution 1 : 2000; Zymed, San Francisco, CA, USA) for 1 hour. The unbound antibodies were removed by washing and bound antibodies were detected by incubation with 3,3',5,5'-tetramethylbenzidine substrate for 30 minutes. After the reaction was stopped by $1 \mathrm{M}$ sulfuric acid, the resultant color reaction was read at $450 \mathrm{~nm}$ using a Victor2 apparatus (Perkin Elmer Life Sciences, Turku, Finland). ${ }^{13)}$

\section{Coronary angiography}

Coronary angiography was performed through the right radial artery, or, if this approach was considered difficult, through the right femoral artery. Obstructive CAD was defined as a reduction of 50\% or more in the luminal diameter of at least one major epicardial cor- onary artery, as determined by quantitative coronary angiography analysis. The severity of CAD was determined by the number of diseased vessels with obstructive CAD and identified by the modified Gensini scoring system, which is used to reflect the extent of coronary atherosclerosis by calculating the score based on the number of stenotic coronary artery segments, the degree of their lumen stenosis, and the localization of stenotic changes. ${ }^{14)}$

\section{Brachial-ankle pulse wave velocity}

All subjects abstained from caffeine-containing food or drink for at least 45 minutes prior to measurement of baPWV. After a subject had been resting in the supine position for at least 5 minutes in a quiet room, blood pressure and baPWV were measured using a VP1000 automated waveform analyzer (Colin Medical Instruments, Komaki, Japan). Blood pressure was measured in both arms with the blood pressure cuffs of the device, and the higher value was used for the analysis. The highest value of baPWV measured on either side of each patient was used for the analysis.

\section{Statistical analyses}

Values are expressed as mean $\pm S D$. Comparison of the continuous variables between subgroups was conducted via unpaired t-test and one-way analysis of variance test, and categorical variables between subgroups was conducted via chi-square test. Correlations were assessed using Pearson's correlation test. Receiver operating characteristic (ROC) curve analysis was used to determine the optimal cutoff point of the serum levels of AGEs for predicting obstructive CAD. Multiple regression analysis using serum levels of AGEs, creatinine, and age as independent variables significantly different in univariate analysis were performed for the presence of obstructive CAD and the associations with the number of vessel diseases (VDs). All analyses were conducted using the Statistical Package for the Social Sciences (SPSS) statistical package ver. 15 (SPSS Inc., Chicago, IL, USA) and $p<0.05$ was considered to be significant.

\section{Results}

\section{Baseline characteristics}

The clinical characteristics of the patients in this study are shown in Table 1. This study included 44 diabetics (65 49 years, 59\% male)

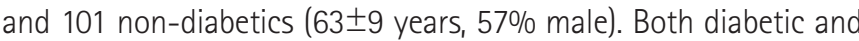
non-diabetic individuals were classified into three subgroups based on the number of diseased vessels with obstructive CAD: 0, 1, and more than 2 VDs and calculated using the modified Gensini scoring system. Among these subgroups, there were significant differences in age, serum levels of creatinine, and AGEs in diabetic pa- 
Table 1. Clinical characteristics of the study subjects

\begin{tabular}{|c|c|c|c|c|c|c|c|c|c|c|}
\hline & \multicolumn{4}{|c|}{ Non-diabetes patients $(n=101)$} & \multirow{4}{*}{ p } & \multirow{2}{*}{\multicolumn{4}{|c|}{$\begin{array}{l}\text { Diabetic patients }(n=44) \\
\text { neer of obstructive coronary arteries }\end{array}$}} & \multirow{4}{*}{ p } \\
\hline & \multirow[b]{3}{*}{$0(n=49)$} & of obstructi & ive coronary & arteries & & & & & & \\
\hline & & \multicolumn{3}{|c|}{ Obstructive CAD } & & \multirow[b]{2}{*}{$0(n=8)$} & \multicolumn{3}{|c|}{ Obstructive CAD } & \\
\hline & & $\begin{array}{c}\text { Total } \\
(n=52)\end{array}$ & $\begin{array}{c}1 \\
(n=23)\end{array}$ & $\begin{array}{c}\geq 2 \\
(n=29)\end{array}$ & & & $\begin{array}{c}\text { Total } \\
(n=36)\end{array}$ & $\begin{array}{c}1 \\
(n=9)\end{array}$ & $\begin{array}{c}\geq 2 \\
(n=27)\end{array}$ & \\
\hline Men $(\%)$ & $23(22.8)$ & $35(35)$ & $11(11)$ & $24(24)$ & 0.005 & $3(6.8)$ & $23(52)$ & $6(14)$ & $17(39)$ & 0.382 \\
\hline Age (years) & $61 \pm 9$ & $64 \pm 8$ & $62 \pm 9$ & $65 \pm 8$ & 0.129 & $59 \pm 8$ & $66 \pm 9$ & $60 \pm 6$ & $68 \pm 9^{\ddagger}$ & 0.016 \\
\hline $\mathrm{SBP}(\mathrm{mm} \mathrm{Hg})$ & $135 \pm 20$ & $139 \pm 28$ & $136 \pm 35$ & $141 \pm 21$ & 0.611 & $128 \pm 21$ & $142 \pm 29$ & $154 \pm 42$ & $138 \pm 23$ & 0.254 \\
\hline $\mathrm{DBP}(\mathrm{mm} \mathrm{Hg})$ & $77 \pm 13$ & $79 \pm 12$ & $79 \pm 14$ & $80 \pm 11$ & 0.676 & $80 \pm 17$ & $71 \pm 14$ & $80 \pm 14$ & $68 \pm 13$ & 0.110 \\
\hline Heart rate (bpm) & $61 \pm 14$ & $62 \pm 14$ & $66 \pm 18$ & $59 \pm 9$ & 0.215 & $69 \pm 18$ & $65 \pm 11$ & $66 \pm 15$ & $65 \pm 11$ & 0.677 \\
\hline Hypertension (\%) & $20(20)$ & $33(33)^{\ddagger}$ & $18(18)$ & $15(15)$ & 0.012 & $5(11)$ & $26(59)$ & $7(16)$ & $19(43)$ & 0.789 \\
\hline Duration of DM (years) & - & - & - & - & - & $4.5 \pm 6.2$ & $6.8 \pm 6.9$ & $5.5 \pm 4.7$ & $7.2 \pm 7.6$ & 0.644 \\
\hline Smoker (\%) & $13(15)$ & $30(35)^{*}$ & $10(12)$ & $20(23)$ & $<0.001$ & $2(5)$ & $17(46)$ & $5(14)$ & $12(32)$ & 0.379 \\
\hline Serum creatinine $(\mathrm{mg} / \mathrm{dL})$ & $0.86 \pm 0.24$ & $0.94 \pm 0.25$ & $0.83 \pm 0.25$ & $1.03 \pm 0.21$ & 0.003 & $0.68 \pm 0.20$ & $0.89 \pm 0.19^{*}$ & $0.87 \pm 0.25$ & $0.89 \pm 0.16^{\ddagger}$ & 0.024 \\
\hline Total cholesterol (mg/dL) & $171 \pm 34$ & $182 \pm 37$ & $185 \pm 37$ & $179 \pm 37$ & 0.272 & $177 \pm 40$ & $165 \pm 45$ & $147 \pm 35$ & $171 \pm 47$ & 0.299 \\
\hline Triglycerides (mg/dL) & $119 \pm 56$ & $133 \pm 54$ & $122 \pm 38$ & $141 \pm 63$ & 0.251 & $118 \pm 36$ & $158 \pm 105$ & $219 \pm 173$ & $137 \pm 60$ & 0.053 \\
\hline $\mathrm{HDL}-\mathrm{C}(\mathrm{mg} / \mathrm{dL})$ & $52 \pm 12$ & $48 \pm 11$ & $48 \pm 12$ & $49 \pm 11$ & 0.336 & $49 \pm 6$ & $49 \pm 12$ & $41 \pm 8$ & $51 \pm 12$ & 0.075 \\
\hline LDL-C (mg/dL) & $97 \pm 31$ & $106 \pm 28$ & $113 \pm 26$ & $103 \pm 30$ & 0.649 & $118 \pm 27$ & $100 \pm 35$ & $81 \pm 44$ & $108 \pm 31$ & 0.397 \\
\hline FBS (mg/dL) & $97 \pm 11$ & $99 \pm 13$ & $99 \pm 13$ & $99 \pm 12$ & 0.805 & $142 \pm 25$ & $125 \pm 31$ & $140 \pm 46$ & $121 \pm 26$ & 0.167 \\
\hline HbA1c (\%) & $5.7 \pm 0.4$ & $5.8 \pm 0.4$ & $5.7 \pm 0.3$ & $5.8 \pm 0.5$ & 0.666 & $7.2 \pm 0.9$ & $7.1 \pm 1.1$ & $7.3 \pm 1.9$ & $7.0 \pm 0.7$ & 0.751 \\
\hline AGEs (mU/mL) & $1.90 \pm 0.34$ & $1.86 \pm 0.39$ & $1.98 \pm 0.45$ & $1.76 \pm 0.32$ & 0.096 & $1.85 \pm 0.29$ & $2.16 \pm 0.29^{*}$ & $1.96 \pm 0.18$ & $2.23 \pm 0.29^{* \dagger}$ & 0.002 \\
\hline BaPWV (cm/s) & $1622 \pm 464$ & $1612 \pm 302$ & $1599 \pm 317$ & $1621 \pm 295$ & 0.970 & $1600 \pm 289$ & $1746 \pm 385$ & $1560 \pm 263$ & $1808 \pm 403$ & 0.132 \\
\hline Modified Gensini score & $3.0 \pm 3.9$ & $38.7 \pm 51.5^{*}$ & $14.8 \pm 10.2$ & $60.4 \pm 63.6^{* t}$ & $<0.001$ & $6.1 \pm 6.8$ & $53.6 \pm 57.5^{\ddagger}$ & $11.4 \pm 6.9$ & $65.0 \pm 59.8^{t \neq}$ & 0.004 \\
\hline
\end{tabular}

Data are expressed as $n(\%)$ or mean \pm SD. Differences between groups were tested by chi-square and ANOVA with Bonferroni's post hoc test performed if $p<0.05 .{ }^{*} p<0.01$ vs. 0 -vessel disease, ${ }^{+} p<0.05$ vs. 1-vessel disease, ${ }^{\dagger} p<0.05$ vs. 0 -vessel disease. CAD: coronary artery disease, SBP: systolic blood pressure, DBP: diastolic blood pressure, DM: diabetes mellitus, HDL-C: high density lipoprotein-cholesterol, LDL-C: low density lipoprotein-cholesterol, FBS: fasting blood sugar, HbA1c: hemoglobin A1c, AGEs: advanced glycation end-products, baPWV: brachial-ankle pulse wave velocity, ANOVA: analysis of variance

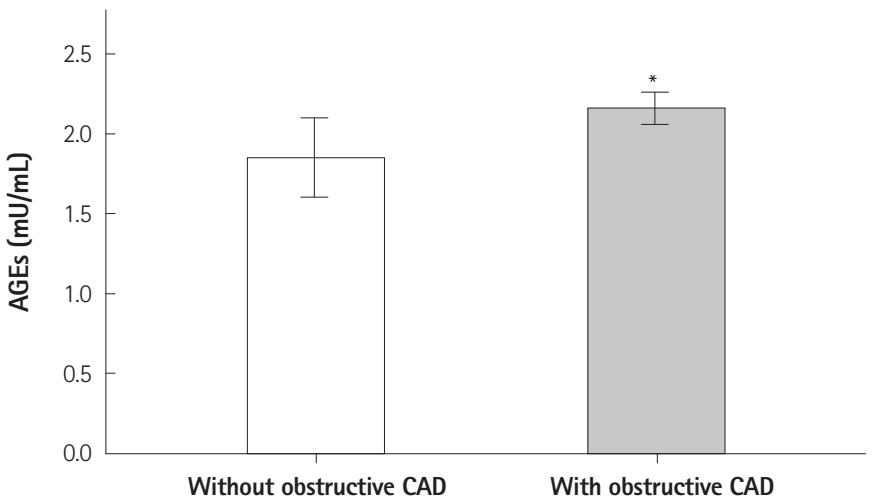

Fig. 1. Comparison of serum levels of AGEs with and without obstructive $C A D$ in diabetic patients. ${ }^{*} p=0.010$. AGEs: advanced glycation end-products, CAD: coronary artery disease.

tients and, for sex, incidence of hypertension and smoking, serum levels of creatinine in non-diabetic patients. Modified Gensini score differed significantly among subgroups classified by the number of VDs in both diabetics and non-diabetics.

\section{Serum advanced glycation end-products and obstructive coronary artery disease}

Serum levels of AGEs were significantly higher in diabetics with obstructive CAD than in those without obstructive CAD $(2.16 \pm 0.29$ vs. $1.85 \pm 0.29 \mathrm{mU} / \mathrm{mL}, \mathrm{p}=0.010$ ) (Fig. 1), but did not differ significantly between non-diabetics with and without obstructive CAD (1.86 \pm 0.39 vs. $1.90 \pm 0.34 \mathrm{mU} / \mathrm{mL}$, respectively; $p=0.590$ ). In the ROC analysis to determine the optimal cut-off value of serum levels of AGEs as a predictor of obstructive CAD in diabetics, the cut-off value of $1.98 \mathrm{mU} / \mathrm{mL}$ had $64 \%$ sensitivity and 63\% specificity in predicting obstructive CAD ( $p=0.031$ ) (Fig. 2).

Serum levels of AGEs were significantly correlated with the number of $V D$ in diabetics $(r=0.504, p<0.001)$ (Fig. 3), but they were not significantly correlated with the number of VDs in non-diabetic patients $(r=-0.136, p=0.174)$.

Multiple regression analysis suggested that serum levels of AGEs and creatinine could independently predict obstructive $C A D$, and that serum levels of AGEs were associated with the number of VDs in diabetics (Table 2). 


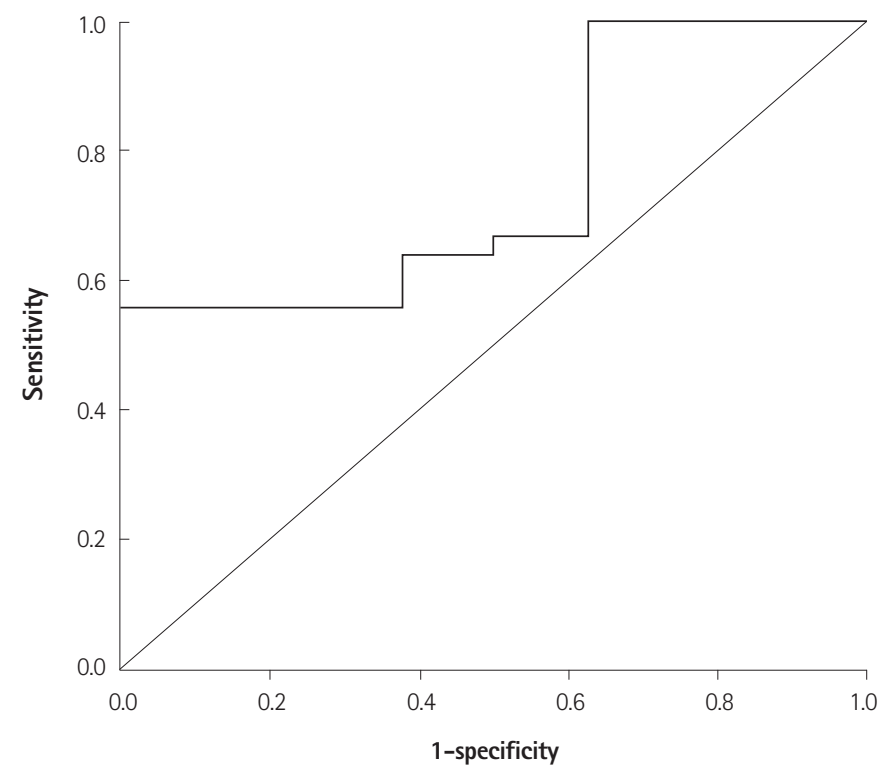

Fig. 2. Receiver operating characteristics curve of serum levels of AGEs for obstructive CAD in diabetic patients. $A \cup C=0.747 ; 95 \% \mathrm{Cl} 0.576$ to 0.917 ; $\mathrm{p}=0.031$. The cut-off point for levels of AGEs using the Youdean index was $1.98 \mathrm{mU} / \mathrm{mL}$ (sensitivity=0.639, specificity=0.625). AGEs: advanced glycation end-products, CAD: coronary artery disease. AUC: area under the curve, $\mathrm{Cl}$ : confidence interval.

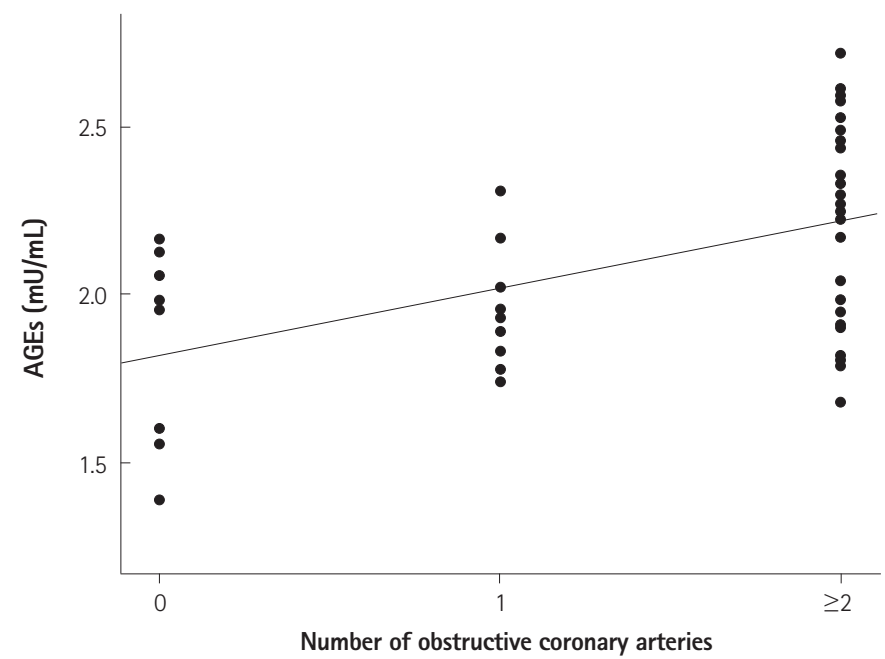

Fig. 3. Correlation between serum levels of AGEs and the number of diseased vessels with obstructive coronary artery disease in diabetes patients. $r=0.504$; $p<0.001$. AGEs: advanced glycation end-products.

\section{Serum advanced glycation end-products and brachial-ankle pulse wave velocity}

Serum levels of AGEs were not significantly correlated with baP$W V$ in diabetics $(r=0.178, p=0.247)$ or non-diabetics $(r=-0.113, p=$ 0.259 ). BaPWW was also not significantly different in diabetics (1746 \pm 385 vs. $160 \pm 289 \mathrm{~cm} / \mathrm{s}$, respectively; $\mathrm{p}=0.320$ ) or non-diabetics (1612 \pm 302 vs. $1622 \pm 464 \mathrm{~cm} / \mathrm{s}$, respectively; $p=0.893$ ), with or without obstructive CAD.
Table 2. Multiple regression analysis for the presence of obstructive CAD and the number of VDs in diabetics

\begin{tabular}{lcccccccc}
\hline & \multicolumn{3}{c}{$\begin{array}{c}\text { Presence of obstructive } \\
\text { CAD }\end{array}$} & & & \multicolumn{3}{c}{ Number of VDs } \\
\cline { 2 - 4 } \cline { 7 - 9 } & B & SE & p & & B & SE & p \\
\hline Age & - & - & - & & 0.022 & 0.011 & 0.053 \\
Serum creatinine & 0.625 & 0.268 & 0.025 & & 0.847 & 0.506 & 0.102 \\
AGEs & 0.374 & 0.175 & 0.038 & & 0.975 & 0.332 & 0.005
\end{tabular}

B: standard regression cofficient, CAD: coronary artery disease, VDs: vessel diseases, AGEs: advanced glycation end-products, SE: standard error

\section{Serum advanced glycation end-products and other laboratory data}

Serum levels of AGEs were not significantly correlated with hemoglobin A1c (HbA1c) $(r=-0.147, p=0.347)$ and serum creatinine $(r=0.256$, $\mathrm{p}=0.093)$ in diabetics.

\section{Discussion}

Diabetes is closely associated with macrovascular and microvascular disease. As a potential pathophysiologic mechanism for vascular complications related to diabetes, recent studies have focused on the role of AGEs, a biochemically diverse group of compounds that accumulate with aging, and according to the status of renal impairment and diabetic state.

Advanced glycation end-products-mediated intramolecular and intermolecular cross-linking of collagen in the arterial wall lead to a decrease in enzymatic proteolysis and degradation rate by altering its surface charge and packing density, ultimately reducing arterial compliance. ${ }^{15) 16)}$ Increased arterial stiffness and decreased vascular distensibility leads to increased systolic pressure and cardiac afterload, and decreased diastolic blood pressure that could potentially compromise coronary blood flow. ${ }^{788}$ Increased arterial stiffness is reflected in increased PWV, and is known to be a predictor of coronary heart disease, stroke, and cardiovascular mortality. ${ }^{1718)}$

There are many recognized binding proteins for AGEs, some of which act as receptors, such as AGE receptors 1, 2, and 3, macrophage scavenger receptors 1 and 2, CD 34, megalin, ezrin, radixine, moesin protein, and RAGE. AGEs contribute to the impairment of endothelial function by reducing nitric oxide ${ }_{1}^{19)}$ increasing the oxidation of low density lipoprotein ${ }_{1}^{20)}$ chemotaxis and activation of monocytes, ${ }_{1}^{21)}$ increasing generation of reactive oxygen species, ${ }^{22)}$ upregulating inflammation, ${ }_{1}^{23)}$ and, finally, lead to the development of atherosclerosis via these receptors. ${ }^{24)}$ Recently, the RAGE receptor has been recognized as being most biologically significant in the setting of atherosclerosis.

Previous studies reported serum levels of AGEs to be related to the degree of coronary atherosclerosis in both diabetics ${ }^{910)}$ and non- 


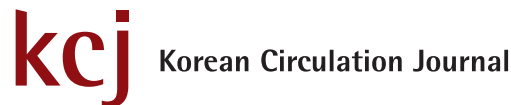

diabetics ${ }^{11)}$ with CAD. Recently, Semba et al. ${ }^{12)}$ reported that increased serum levels of AGEs were associated with increased arterial stiffness among relatively healthy community-dwelling adults. This study evaluated the usefulness of serum levels of AGEs in predicting obstructive $C A D$, and the association between serum levels of AGEs and arterial stiffness using baPWV, the most widely-used PWV index, in both diabetic and non-diabetic patients suspected of having CAD. We postulated that serum levels of AGEs are useful in predicting the presence of $C A D$, and that is associated with increased arterial stiffness in patients suspected of having CAD. Our major finding was that serum levels of AGEs were significantly higher in diabetic patients with obstructive CAD than in those without obstructive $C A D$, and reflected the severity of coronary atherosclerosis irrespective of arterial stiffness as measured by baPWV in diabetic patients. Although a trend of increasing arterial stiffness in diabetic patients with obstructive CAD was noted, baPWV was not correlated with serum levels of AGEs or showed significant difference between the absence and presence of CAD in diabetes. This may suggest that the receptor dependent actions of AGEs, through receptors such as RAGE, tend to be more potent than increased arterial stiffness in the genesis of coronary atherosclerotic disease, and also suggests that an increase in serum AGEs more precisely reflects the progression of atherosis than increased arterial wall stiffness in patients with diabetes. Some of the increase in serum AGEs may also represent increased modification of low density lipoprotein as a result of glycation, which plays a central role in the development of atherosclerosis. ${ }^{25)}$ Although a PWV is good method to predict cardiovascular events, evaluation of PWV and serum levels of AGEs together may be more effective to predict CAD in diabetic patients, especially within normal range of PWV value. Additionally, it is important to note that serum levels of AGEs are not increased with obstructive CAD or reflect the severity of coronary atherosclerosis in non-diabetic patients. This result may support the critical role for AGEs in the pathogenesis of coronary atherosclerosis in diabetic individuals.

Unlike Semba et al. ${ }^{12)}$ who reported that increased serum levels of AGEs were associated with increased arterial stiffness, our finding did not showed significant association between serum AGEs and baPWV in both diabetics and non-diabetics. This might be caused by the difference in study population. The aforementioned study examined relatively healthy community-dwelling adults. By comparison, our study population was high-risk patients who had been referred to a tertiary referral hospital to be evaluated for suspected CAD. It might be also interpreted that increased serum levels of AGEs might more accurately reflect the progression of coronary atherosis than increased arterial stiffness in diabetic patients with suspected of having CAD.
Previous studies have suggested that chronic hyperglycemia contributes to increased cardiovascular risk, and one potential mechanism may be through the formation of AGEs. ${ }^{3-5)}$ However, Ono et al. ${ }^{26)}$ found that serum levels of AGEs did not correlate with serum $\mathrm{HbA} 1 \mathrm{C}$ in patients whose serum levels of creatinine were $<4.0 \mathrm{mg} /$ $\mathrm{dL}$. Kilhovd et al. ${ }^{9)}$ also reported a lack of significant correlation between serum AGEs and HbA1c. Kiuchi et al. ${ }^{10)}$ found that serum levels of AGEs were not correlated with recent serum $\mathrm{HbA} 1 \mathrm{C}$, but were positively correlated with serum mean 4-year $\mathrm{HbA} 1 \mathrm{C}$. We also found a lack of significant correlation between serum levels of AGEs and serum $\mathrm{HbA} 1 \mathrm{C}$ in diabetics. This was probably caused by a different turnover and a time lag between the production of AGEs and removal from tissue AGEs accumulation, and may suggest that long term poor glycemic control is associated with AGEs production.

Renal function affects serum levels of AGEs. Makita et al. ${ }^{27)}$ reported that serum levels of AGEs were increased in end stage renal disease but not in normal renal function. In our study, only two patients had decreased renal function (serum levels of creatinine $>1.5$ $\mathrm{mg} / \mathrm{dL}$ ), and there were no end stage renal disease patients. Multiple regression analysis demonstrated that serum creatinine is related to the presence of obstructive CAD, but not independently associated with the number of VDs.

There were some limitations to this study. The accurate indicator reflecting central arterial stiffness has been considered to be aortic PWV measured as the carotid-femoral PWV (cfPWV). In this study, however, we used the baPWV, which has been increasingly used as an index of arterial stiffness, because the automatic waveform analyzer did not provide the value of cfPWV. A baPWV is indirectly estimated using PWV from the heart to the brachial and posterior tibial arteries, because the pulse wave does not propagate directly from the brachial arteries to the posterior tibial arteries in the same arterial tree. Also, the peripheral arteries do not represent aging and disease-related changes as do the central arteries. Despite these limitations of baPWV, previous studies have suggested that baPWV is a useful measure for arterial stiffness. Yamashina et al. ${ }^{28)}$ reported that baPWV could able to be considered an acceptable marker with an efficacy comparable to that of cfPWV. Sugawara et al. ${ }^{29)}$ also reported that baPWV provide similar qualitative information, which was derived from central arterial stiffness. Thus, it may be reasonable to use baPWV for evaluation of central arterial stiffness in this study. Another limitation of this study is that we did not evaluate other structurally-identified AGEs such as pentosidine, which could be associated with $C A D$, and the RAGE receptor, which is known to contribute significantly to the development of atherosclerosis in both diabetic and non-diabetic subjects.

In summary, the serum level of AGE is an independent predictor of obstructive $C A D$, and reflect the severity of coronary atheroscle- 
rosis irrespective of arterial stiffness only in diabetic patients with suspected CAD. Evaluation of PWV and serum AGEs together may be more effective to identify the risk of CAD in diabetic patients.

\section{Acknowledgments}

This study was supported by a grant from the Korean Health Technology R\&D Project, Ministry for Health \& Welfare, Korea (A102064).

\section{References}

1. Kannel WB, McGee DL. Diabetes and cardiovascular disease: the Framingham study. JAMA 1979;241:2035-8.

2. Haffner SM, Lehto S, Rönnemaa T, Pyörälä K, Laakso M. Mortality from coronary heart disease in subjects with type 2 diabetes and in nondiabetic subjects with and without prior myocardial infarction. $N$ Engl J Med 1998;339:229-34.

3. Turner RC, Millns $H$, Neil HA, et al. Risk factors for coronary artery disease in non-insulin dependent diabetes mellitus: United Kingdom Prospective Diabetes Study (UKPDS: 23). BMJ 1998;316:823-8.

4. De Vegt $F_{1}$ Dekker JM, Ruhé $H G$, et al. Hyperglycaemia is associated with all-cause and cardiovascular mortality in the Hoorn population: the Hoorn Study. Diabetologia 1999;42:926-31.

5. Schleicher ED, Wagner $E_{1}$ Nerlich AG. Increased accumulation of the glycoxidation product N(epsilon)-(carboxymethyl)lysine in human tissues in diabetes and aging. J Clin Invest 1997;99:457-68.

6. Reddy GK. AGE-related cross-linking of collagen is associated with aortic wall matrix stiffness in the pathogenesis of drug-induced diabetes in rats. Microvasc Res 2004;68:132-42.

7. Sutton-Tyrrell K, Najjar SS, Boudreau RM, et al. Elevated aortic pulse wave velocity, a marker of arterial stiffness, predicts cardiovascular events in well-functioning older adults. Circulation 2005;111:3384-90.

8. Mattace-Raso FU, van der Cammen TJ, Hofman A, et al. Arterial stiffness and risk of coronary heart disease and stroke: the Rotterdam Study. Circulation 2006;113:657-63.

9. Kilhovd BK, Berg TJ, Birkeland KI, Thorsby P, Hanssen KF. Serum levels of advanced glycation end products are increased in patients with type 2 diabetes and coronary heart disease. Diabetes Care 1999;22:1543-8.

10. Kiuchi K, Nejima J, Takano T, Ohta M, Hashimoto H. Increased serum concentrations of advanced glycation end products: a marker of coronary artery disease activity in type 2 diabetic patients. Heart 2001;85: 87-91.

11. Kanauchi M, Tsujimoto N, Hashimoto T. Advanced glycation end products in nondiabetic patients with coronary artery disease. Diabetes Care 2001;24:1620-3.

12. Semba RD, Najjar SS, Sun K, Lakatta EG, Ferrucci L. Serum carboxymethyl-lysine, an advanced glycation end product, is associated with increased aortic pulse wave velocity in adults. Am J Hypertens 2009; 22:74-9.

13. Choi EY, Kwon HM, Ahn CW, et al. Serum levels of advanced glycation end products are associated with in-stent restenosis in diabetic patients. Yonsei Med J 2005;46:78-85.
14. Tarchalski J, Guzik P, Wysocki H. Correlation between the extent of coronary atherosclerosis and lipid profile. Mol Cell Biochem 2003;246: 25-30.

15. Tanaka S, Avigad G, Brodsky B, Eikenberry EF. Glycation induces expansion of the molecular packing of collagen. J Mol Biol 1988;203: 495-505.

16. Tsilibary EC, Charonis AS, Reger LA, Wohlhueter RM, Furcht LT. The effect of nonenzymatic glucosylation on the binding of the main noncollagenous NC1 domain to type IV collagen. J Biol Chem 1988;263: 4302-8.

17. Boutouyrie $P_{1}$ Tropeano Al, Asmar $R$, et al. Aortic stiffness is an independent predictor of primary coronary events in hypertensive patients: a longitudinal study. Hypertension 2002;39:10-5.

18. Najjar SS, Scuteri A, Lakatta EG. Arterial aging: is it an immutable cardiovascular risk factor? Hypertension 2005;46:454-62.

19. Bucala R, Tracey KJ, Cerami A. Advanced glycosylation products quench nitric oxide and mediate defective endothelium-dependent vasodilatation in experimental diabetes. J Clin Invest 1991;87:432-8.

20. Bucala R, Makita Z, Vega G, et al. Modification of low density lipoprotein by advanced glycation end products contributes to the dyslipidemia of diabetes and renal insufficiency. Proc Natl Acad Sci U S A 1994; 91:9441-5

21. Kirstein M, Brett J, Radoff S, Ogawa S, Stern D, Vlassara H. Advanced protein glycosylation induces transendothelial human monocyte chemotaxis and secretion of platelet-derived growth factor: role in vascular disease of diabetes and aging. Proc Natl Acad Sci U S A 1990;87: 9010-4.

22. Yan SD, Schmidt AM, Anderson GM, et al. Enhanced cellular oxidant stress by the interaction of advanced glycation end products with their receptors/binding proteins. J Biol Chem 1994;269:9889-97.

23. Basta G. Receptor for advanced glycation endproducts and atherosclerosis: from basic mechanisms to clinical implications. Atherosclerosis 2008;196:9-21.

24. Wautier MP, Chappey O, Corda S, Stern DM, Schmidt AM, Wautier JL. Activation of NADPH oxidase by AGE links oxidant stress to altered gene expression via RAGE. Am J Physiol Endocrinol Metab 2001; 280:E685-94.

25. Bucala R, Makita Z, Koschinsky T, Cerami A, Vlassara H. Lipid advanced glycosylation: pathway for lipid oxidation in vivo. Proc Natl Acad Sci U SA 1993;90:6434-8.

26. Ono Y, Aoki S, Ohnishi K, Yasuda T, Kawano K, Tsukada Y. Increased serum levels of advanced glycation end-products and diabetic complications. Diabetes Res Clin Pract 1998;41:131-7.

27. Makita Z, Radoff S, Rayfield EJ, et al. Advanced glycosylation end products in patients with diabetic nephropathy. N Engl J Med 1991; 325:836-42.

28. Yamashina A, Tomiyama $H_{1}$ Takeda $K$, et al. Validity, reproducibility, and clinical significance of noninvasive brachial-ankle pulse wave velocity measurement. Hypertens Res 2002;25:359-64.

29. Sugawara J, Hayashi K, Yokoi T, et al. Brachial-ankle pulse wave velocity: an index of central arterial stiffness? J Hum Hypertens 2005;19: 401-6. 